Apidologie, 1983, 14 (3), 175-182.

\title{
DICOFOL-RÄUCHERN VON HONIGBIENEN ZUR BEKÄMPFUNG DER MILBE VARROA JACOBSONI
}

O. Van LAERE (1), M. IFANTIDIS (2) und L. DE WAEL (1)

ZUSAMMENFASSUNG

Mit Dicofol und einer kleinen Dosis $\mathrm{KNO}_{3}$ wurden Räucherstreifen mit je $0,5 \mathrm{~g}$ Wirkstoff gefertigt. Unter Verwendung des Rauchapparats nach VAN LAERE et al. (1982) wurden durch Varroa-Milben befallene Bienen - mindestens 10 pro Versuchskäfig bei 3 Wiederholungen - mit Dicofol-Rauch behandelt. Das Räuchern mit $5 \mathrm{mg}$ Dicofol $/ \mathrm{dm}^{3}$ erzielte für Varroa-Milben eine $\mathrm{LD}_{50}$, die um eine Räucherdauer von 9 Minuten lag. Bei $10 \mathrm{mg}$ Dicofol $/ \mathrm{dm}^{3}$ führten alle Behandlungen zu einer 100 prozentigen Abtötung der Varroa-Parasiten, und zwar ab einer Räucherdauer von 4 Minuten. Bei einer solchen Dosis zeigten sich Räucherdauern von $2,4,8,16$ und 32 Minuten unschädlich für die Bienen. Gegenproben mit $\mathrm{KNO}_{3}$ veranlassten keine Abtötung von Varroa-Milben und Bienen. Hohe Rauchkonzentrationen verursachten eine typische, sofortige Reaktion der Parasiten : sie verliessen die Bienen, so dass sie in intensiverer Weise dem Rauch ausgesetzt wurden.

Die Population der Varroa-Milbe befindet sich während des Jahres in zwei voneinander abgegrenzten Mikroumwelten (micro-environment) im Bienenvolk, nämlich a) in den verdeckelten Brutzellen bzw. b) auf den adulten Bienen und auf den freien inneren Flächen des Nestes. In den brutlosen Perioden des Jahres kommen die weiblichen Imagines der Varroa-Milben ausschliesslich auf den erwachsenen Bienen vor. Wenn dagegen die Brutmenge am höchsten ist, lebt fast die ganze Varroapopulation in den verdeckelten Brutzellen (CHOI und WoO, 1973).

Aus der bisherigen Literatur geht hervor, dass die chemische Bekämpfung des lästigen Parasiten nur ausserhalb der verdeckelten Zellen der Bienenbrut und nur bis zu einem gewissen Grad zu erzielen ist. Der Wachsdeckel der Zellen schliesst

(1) Staatsanstalt für Nematologie und Entomologie (C.L.O.-Gent), Van Gansberghelaan 96, B-9220 Merelbeke, Belgien.

(2) Institut für Bienenkunde der Landwirtschaftlichen Fakultät, Universität Thessaloniki, Griechenland. 
den direkten Kontakt der chemischen Mittel mit den Milben aus und die Wirkung der meisten von den verwendeten Varroaziden ist abhängig von der Jahreszeit. Das Kontaktakarizid Dicofol, oder 1,1 bis ( $p$-Chlorophenyl) 2,2,2 Trichloräthanol - auf den Markt gebracht unter dem Namen "Kelthane " $\left({ }^{*}\right)$ - ist schon erfolgreich gegen die Bienenmilbe Acarapis woodi eingesetzt worden (VAN LAERE, 1960).

Für Bestimmungszwecke verwendeten RUTTNER und RITTER (1980) ein für den Gartenbau zusammengesetztes Keltane-Präparat gegen die Varroa-Milbe. Nach der Behandlung verseuchter Völker wurden immer wieder tote Milben auf den Kastenböden gefunden. Die Versuche von RitTer und RuttNer (1980) Kelthane in einer 1,5\% igen Lösung als systemisches Mittel zu verwenden, waren nicht erfolgreich. Das Bespritzen mit einer 1,5\% igen Kelthane-Lösung und der Einsatz einer $6 \%$ igen Kelthane-Mischung in Puderzucker, sowie die Kombination von Kelthane mit Phenothiazin waren nicht befriedigend.

Mit Kelthane-Streupulver erzielte SKRYPNIK (1977), von April bis August, eine Abtötung von Varroa-Parasiten, die höher als $90 \%$ war. Ausserhalb dieser Periode betrug die Abtötungsleistung jedoch nur 40 bis $60 \%$.

FILLIPPOV (1978) hat Bienenvölker ohne befriedigendes Ergebnis mit Dicofol behandelt. Auch Ho, Hsu und AN (1980) haben Dicofol (35\%ige Spritzlösung) in Laborund Praxisversuchen mit analogen Ergebnissen verwendet.

Dieser Beitrag behandelt die Fertigung von Räucherstreifen auf der Basis von Dicofol sowie Laborversuche über die Wirksamkeit dieses Präparats gegen Varroa jacobsoni.

\section{MATERIAL UND METHODEN}

Räucherstreifen auf der Basis von Dicofol (Handelsprodukt "Kelthane » mit 85 \% Wirkstott) werden wie folgt gefertigt :

Stark absorbierendes Papier von $280 \mathrm{~g} / \mathrm{m}^{2}$ wird mit einer 12 gewichtsprozentigen $\mathrm{KNO}_{3}$-Lösung gesättigt und dann waagerecht auf einer nichtabsorbierenden Platte getrocknet (schnell bei $105^{\circ} \mathrm{C}$, oder langsamer bei niedrigeren Temperaturen). Aus dem Papier werden Streifen von $70 \times 45 \mathrm{~mm}$ geschnitten und mit drei Löchern versehen. Eine Anzahl Streifen wird zwischen zwei Metallplatten geirlemmt und, mit den Löchern nach unten, in eine 50 \%ige Dicofol-Azeton-Lösung eingetaucht. Die Lösung befindet sich in einer Schale und steht nur 5 bis $10 \mathrm{~mm}$ hoch, damit die Papierstreifen die Flüssigkeit langsam aufnehmen. Sobald die Dicofol-Lösung bis $10 \mathrm{~mm}$ unter dem Oberrand eingedrungen ist, werden die Streifen entfernt, wonach sie sofort und einzeln bei ca. $75^{\circ} \mathrm{C}$ getrocknet werden. Man bekommt also $0,5 \mathrm{~g}$ Dicofol pro Streifen.

Die Streifen werden im Rauchapparat nach VAN LAERE et al. (1982) verbrannt.

Mit Varroa-Milben besetzte Bienen wurden unmittelbar vor der Behandlung am Bienenstand des Instituts für Bienenkunde der Universität Thessaloniki gesammelt.

(*) Erzeugnis der Rohm und Haas Co, Mailand, Italien. 
Zum Räuchern werden die befallenen Bienen in kleinen Kunststoffkäfigen untergebracht (Abb. 1). Durch die $2 \mathrm{~mm}$ breiten Maschen kann der Rauch ungehindert in die Käfige eindringen. 1 bis $2 \mathrm{~mm}$ über dem Boden des Käfigs befindet sich eine Gazesperre mit Maschen von $1.5 \mathrm{~mm}$. Auf den Boden gefallene Varroa-Milben, die sich eventuell nach einiger Zeit erholen, können sich also wieder an den im Käfig befindlichen Bienen anklammern : sie müssen nur 1 bis $2 \mathrm{~mm}$ hoch kriechen, um über die Gazesperre zu gelangen. Diese Vorrichtung ermöglicht das regelmässige Zählen von toten Milben am Boden. Eine Vertiefung im Deckel enthält Zuckerteig. Nach dem Räuchern wird eine Papierhülse derart über den Käfig geschoben, das alle Öffnungen abgesperrt sind und keine Milben entfliehen können.

Zum Dosieren des Rauches wird ein Kasten aus Perspex von $19.87 \mathrm{dm}^{3}$ verwendet (Abb. I). Der Deckel wird zuerst durch eine Kunststoffolie mit einer Öfnung von $20 \times 9 \mathrm{~cm}$ ersetzt. Der Deckel wird derart auf die Folie gelegt, dass der Rauchapparat auf eine der Ecken gestellt werden kann. Alsdann wird ein Rauchstreifen angezündet und ganz oder teilweise verbrannt. Nach Entfernen des Rauchapparats wird der Perspex-Deckel richtig über der Kunststoffolie angebracht. Der Perspex-Kasten wird dann dreimal langsam umgedreht, so dass sowohl eine ganz gleichmässige wie reproduzierbare Verteilung der Rauchmenge erzielt wird.

Durch Vorversuche wurde der günstigste Bereich der Rauchkonzentration bestimmt. Zwei Konzentrationsgrade von Dicofol Rauch wurden zuletzt geprüft, nämlich $5 \mathrm{mg}$ bzw. $10 \mathrm{mg}$ pro $1 \mathrm{dm}^{3}$. Diese Konzentrationen wurden erreicht, indem jeweils $1 / 4$ bzw. 1/2 Dicofol-Streifen in dem Raum des hier verwendeten Kastens verbrannt wurden. Bei jedem Konzentrationsgrad von Dicofol wurden entsprechend drei bzw. fünf Beräucherungszeiten getestet. Insgesamt wurden also 8 verschiedene Kombinationen von Rauchkonzentration und Zeitdauer probiert.

Bei jeder Kombination wurden 30 mit mindestens einer Varroa-Milbe behaftete Bienen behandelt. Die Tiere von beiden Versuchsansätzen wurden in drei gleich starke Gruppen geteilt und in drei Käfige gebracht.

Die Käfige werden senkrecht auf den Behandlungstisch (E : Abb. 1) gestellt, während die Behandlungskammer (A : Abb. 1) mit gleichmässig verteiltem Rauch einer bestimmten Dosierung gefüllt wird. Die gefüllte Kammer wird nun schnell über die Käfige gestellt, womit die Behandlungsdauer anfängt. Die Beräucherungen wurden bei Zimmertemperatur $\left(22-25^{\circ} \mathrm{C}\right)$ durchgeführt. Nach Ablauf der vorher bestimmten Zeit wird die Rauchkammer entfernt, womit die Beobachtungsdauer anfängt. Die von einer varroadichten Papierhülse umgebenen Käfige werden dann in einen Brutschrank bei $34^{\circ} \mathrm{C}$ gestellt. Nach der letzten Beobachtung werden alle Bienen in einer leichten Seifenlösung gewaschen und danach noch einmal individuell auf die Anwesenheit etwaiger Varroa-Milben untersucht.

\section{ERGEBNISSE UND DISKUSSION}

\section{Die Tabellen 1 und 2 zeigen die Daten bezüglich der Mortalität in absoluten} Zahlen von Bienen bzw. von Varroa-Milben nach einer Beräucherung der Versuchstiere mit dem Akarazid Dicofol unter bestimmten Laborbedingungen.

Die Beräucherung mit $5 \mathrm{mg}$ Dicofol $/ \mathrm{dm}^{3}$ für eine Zeitspanne von 32 Minuten führt nach 24 Std. zur drastischen Verminderung der Milbenzahl, nicht aber zum absoluten Abtöten der Milben.

Mit abnehmender Beräucherungszeit wird das Verhältnis Anzahl von toten Milben/anfänglicher Anzahl Varroa pro Gruppe immer kleiner. Bei verdoppelter Konzentration des Akarizids, d.h. bei $10 \mathrm{mg} / \mathrm{dm}^{3}$ und einer Beräucherungszeit von nur 4 min wird ein $100 \%$ iges Abtöten der Versuchsvarroae innerhalb von 24 Std. erreicht. Diese Dosierung ist für die Bienen zugleich unschädlich, wie diejenige welche bei eineı Beräucherungsdauer von 32 min entsteht, was der Vergleich mit der Kontrolle zeigt (Tabelle 2). 
TAB. 1. - Mortalität der Bienen und Varroa-Milben (in absoluten Zahlen) nach Dicofol-Beräucherung mit $5 \mathrm{mg} / \mathrm{dm}^{3}$ und bei variierter Behandlungsdauer.

TABL. 1. - Mortality (in absolute numbers) of bees and Varroa mites after Dicofol-treatment with $5 \mathrm{mg}$ per $\mathrm{dm}^{3}$ and different treatment times.

\begin{tabular}{|c|c|c|c|c|c|c|}
\hline \multirow[t]{2}{*}{$\begin{array}{c}\text { Versuchstiere } \\
\text { Test Object }\end{array}$} & \multirow[t]{2}{*}{$\begin{array}{c}\text { Beräucherungs- } \\
\text { dauer } \\
\text { (in Minuten) } \\
\text { Fumigation time } \\
\text { (in minutes) }\end{array}$} & \multirow[t]{2}{*}{$\begin{array}{l}\text { Anfängliche } \\
\text { Zahl von Tieren } \\
\text { pro Gruppe } \\
\text { Initial number } \\
\text { of animals } \\
\text { per group }\end{array}$} & \multicolumn{4}{|c|}{$\begin{array}{l}\text { Zahl von toten Versuchstieren } \\
\text { nach der Beräucherung } \\
\text { Number of dead animals } \\
\text { after a fumigation time of }\end{array}$} \\
\hline & & & $2 \mathrm{~h}$ & $4 \mathrm{~h}$ & $8 \mathrm{~h}$ & $24 \mathrm{~h}$ \\
\hline Varroa-Milben & 8 & 25 & 3 & 7 & 8 & 12 \\
\hline Varro amites & $\begin{array}{l}16 \\
32\end{array}$ & $\begin{array}{l}30 \\
31\end{array}$ & $\begin{array}{l}0 \\
0\end{array}$ & $\begin{array}{l}4 \\
3\end{array}$ & $\begin{array}{l}17 \\
10\end{array}$ & $\begin{array}{l}20 \\
27\end{array}$ \\
\hline Bienen & 8 & 26 & 0 & 0 & 0 & 0 \\
\hline Bees & $\begin{array}{l}16 \\
32\end{array}$ & $\begin{array}{l}30 \\
30\end{array}$ & $\begin{array}{l}0 \\
1\end{array}$ & $\begin{array}{l}0 \\
1\end{array}$ & $\begin{array}{l}1 \\
1\end{array}$ & $\begin{array}{l}1 \\
1\end{array}$ \\
\hline
\end{tabular}

TAB. 2. - Mortalität (in absoluten Zahlen) der Bienen und der Varroa-Milben nach Dicofol-Beräucherung mit $10 \mathrm{mg} / \mathrm{dm}^{3}$ und bei variierter Behandlungsdauer.

TABL. 2. - Mortality (in absolute numbers) of bees and Varroa mites after Dicofol-treatment with $10 \mathrm{mg} \mathrm{per} \mathrm{dm}^{3}$ and different treatment times.

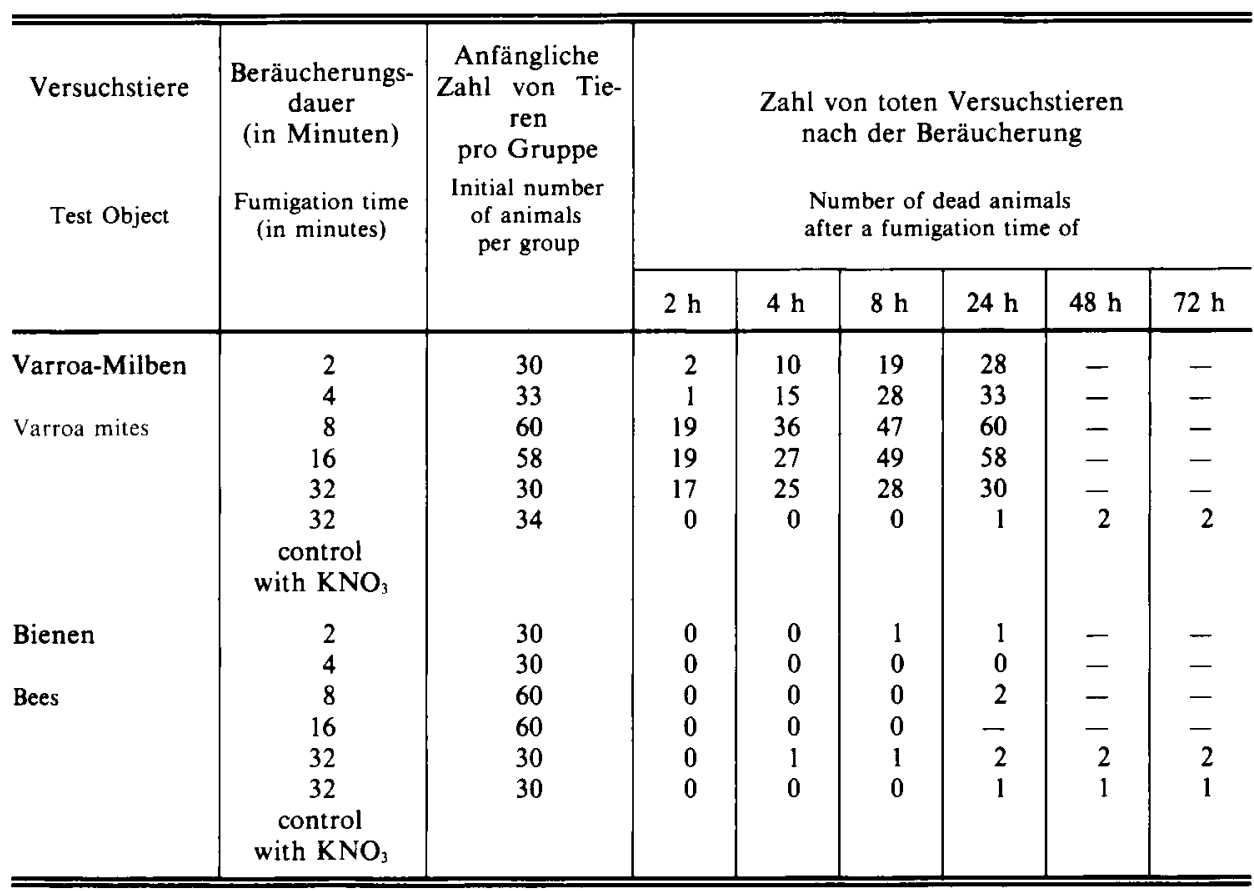


An Hand der Daten von Tabelle 1 ist die $\mathrm{LD}_{50}$ des Dicofols für die Varroa-Milbe errechnet (Abb. 2). Es ist zu ersehen, dass $50 \%$ der Varroa-Milben mit der Dosierung " $5 \mathrm{mg} / 1 \mathrm{dm}^{3} / 9$ minütiger Beräucherungszeit " innerhalb von $24 \mathrm{Std}$. getötet werden. Der Vergleich zwischen den Abbildungen 2 und 3 veranschaulicht noch

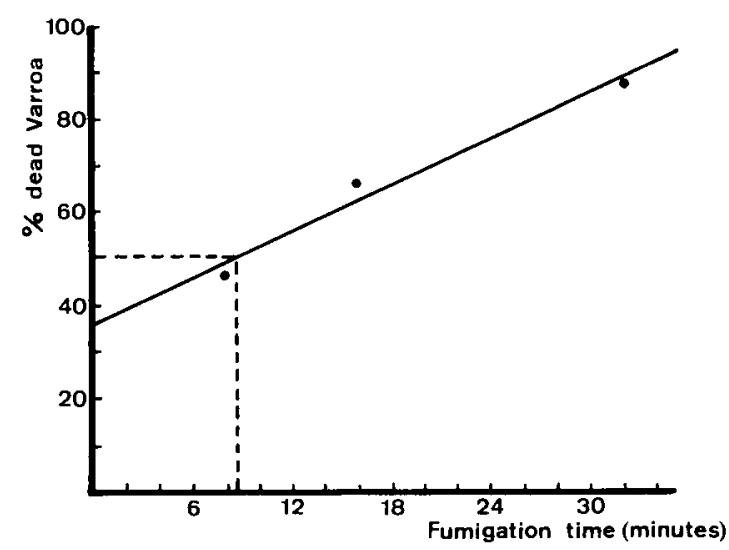

Aвв. 2. - Mortalität der Varroa-Milbe 24 Stunden nach der Beräucherung mit $5 \mathrm{mg}$ Dicofol pro $1 \mathrm{dm}^{3}$.

FIG. 2. - Mortality of Varroa mites, 24 hours after smoke treatment with $5 \mathrm{mg}$ Dicofol per $\mathrm{dm}^{3}$.

deutlicher den Grad der Toxizität des Dicofols gegen die Varroa-Milbe, wenn die Menge des Akarizides von $5 \mathrm{mg}$ zu $10 \mathrm{mg}$ steigt. Innerhalb von $8 \mathrm{Std}$. werden $80 \%$ der Milben durch eine Dosierung « $10 \mathrm{mg} / 1 \mathrm{dm}^{3} / 9$ minütiger Beräucherungszeit » getötet.

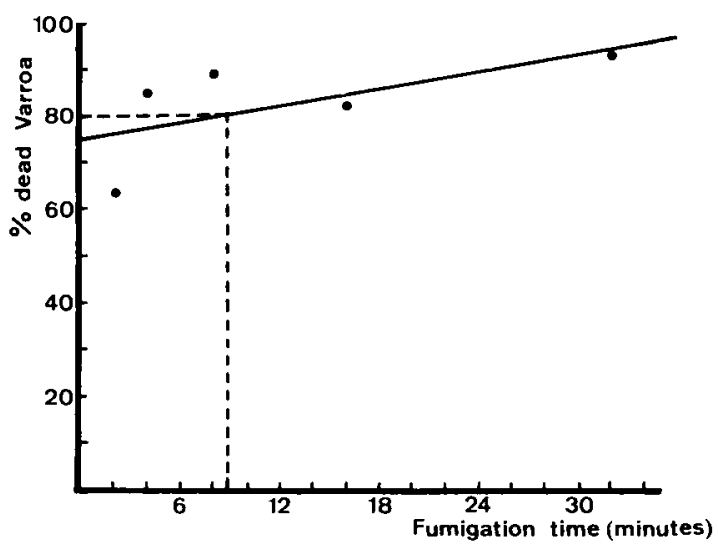

Aвв. 3. - Mortalität der Varroa-Milbe 8 Stunden nach der Beräucherung mit $10 \mathrm{mg}$ Dicofol pro $1 \mathrm{dm}^{3}$.

FIG. 3. - Mortality of Varroa mites, 24 hours after smoke treatment with $10 \mathrm{mg}$ Dicofol per $\mathrm{dm}^{3}$. 
Die Tatsache, dass eine 100 prozentige Abtötung der Parasiten ziemlich leicht erzielt wird, weist in die Richtung einer maximalen Verminderung der Widerstandsfähigkeit gegen Dicofol.

Eine wichtige Beobachtung während der Versuche war, dass die Varroa-Milben bei hohen Rauchkonzentrationen die Bienen sofort verlassen. Diese typische Reaktion während der ersten Minuten der Behandlung hat zur Folge, dass die Parasiten in intensiverer Weise dem Rauch ausgesetzt werden. Auch während der Vorversuche wurde dieses Phänomen wiederholt festgestellt. Es kann also vorausgesetzt werden, dass Behandlungen in der Praxis sowohl bei hohen Rauchkonzentrationen als auch während relativ kurzer Zeiten durchzuführen sind. Dies ist von besonderer Bedeutung für die Ausarbeitung von Behandlungen in der Praxis, wo es vorteilhaft ist, die Sperrdauer der Bienenkästen auf ein Minimum zu reduzieren.

Es kann geschlossen werden, dass das Dicofol-Räuchern von Bienen, verseucht durch Varroa jascobsoni, zu einer 100 prozentigen Abtötung der Parasiten führt, wenn eine Dosis von $10 \mathrm{mg}$ Dicofol $/ \mathrm{dm}^{3}$ angewandt wird und das Räuchern selbst 4 Minuten dauert. Eine solche Dosis zeigt sich unschädlich für die Bienen. Alles deutet darauf hin, dass die ausgearbeiteten Dicofol-Räucherstreifen für die praktische Behandlungen von Bienenvölkern gegen Varroatose geeignet sind.

Weitere Freilandsversuche sind dennoch erforderlich, um die Wirkung von Dicofol auch unter den Bedingungen in der Beute zu prüfen und eventuelle Rückstände im Honig zu bestimmen.

Die hier angewendete Technik zur Bestimmung der Milben- bzw. Bienentoxizität des Dicofols erlaubt die Prüfung der entsprechenden Toxizität anderer Räucherpräparate unter standardisierten Laborbedingungen, um vergleichbare Ergebnisse bekommen zu können.

Eingegangen im Februar 1983. Reçu pour publication en février 1983.

\author{
RÉSUME \\ FUMIGATION DES ABEILLES AU DICOFOL COMME TRAITEMENT \\ CONTRE L'ACARIEN VARROA JACOBSONI
}

Les auteurs ont confectionné des tickets fumigènes à base de dicofol et d'une petite dose de $\mathrm{KNO}_{3}$. Chaque ticket contenait $0,5 \mathrm{~g}$ de substance active. Des abeilles infectées de Varroa -10 abeilles par cagette d'essai en 3 répétitions - ont été traitées à la fumée de dicofol, au moyen de l'appareil de fumigation de VAN LAERE et coll. (1982). La $\mathrm{DL}_{50}$ à l'égard de Varroa se situe à 9 minutes de fumigation à la densité de $5 \mathrm{mg}$ de dicofol au $\mathrm{dm}^{3}$. Avec $10 \mathrm{mg}$ de dicofol au dm $\mathrm{dm}^{3}$, tous les traitements procurent l'extermination à $100 \%$ des parasites à partir de 4 minutes de fumigation. Des fumigations de $2,4,8,16$ et 32 minutes à la même dose sont inoffensives pour les abeilles. Les traitements-témoins au $\mathrm{KNO}_{3}$ n'ont pas provoqué de mortalité parmi les parasites ni parmi les abeilles. De fortes concentrations de fumée déclenchent chez les parasites une réaction typique et immédiate : ils quittent les abeilles et sont ainsi exposés de façon plus intensive à la fumée. 

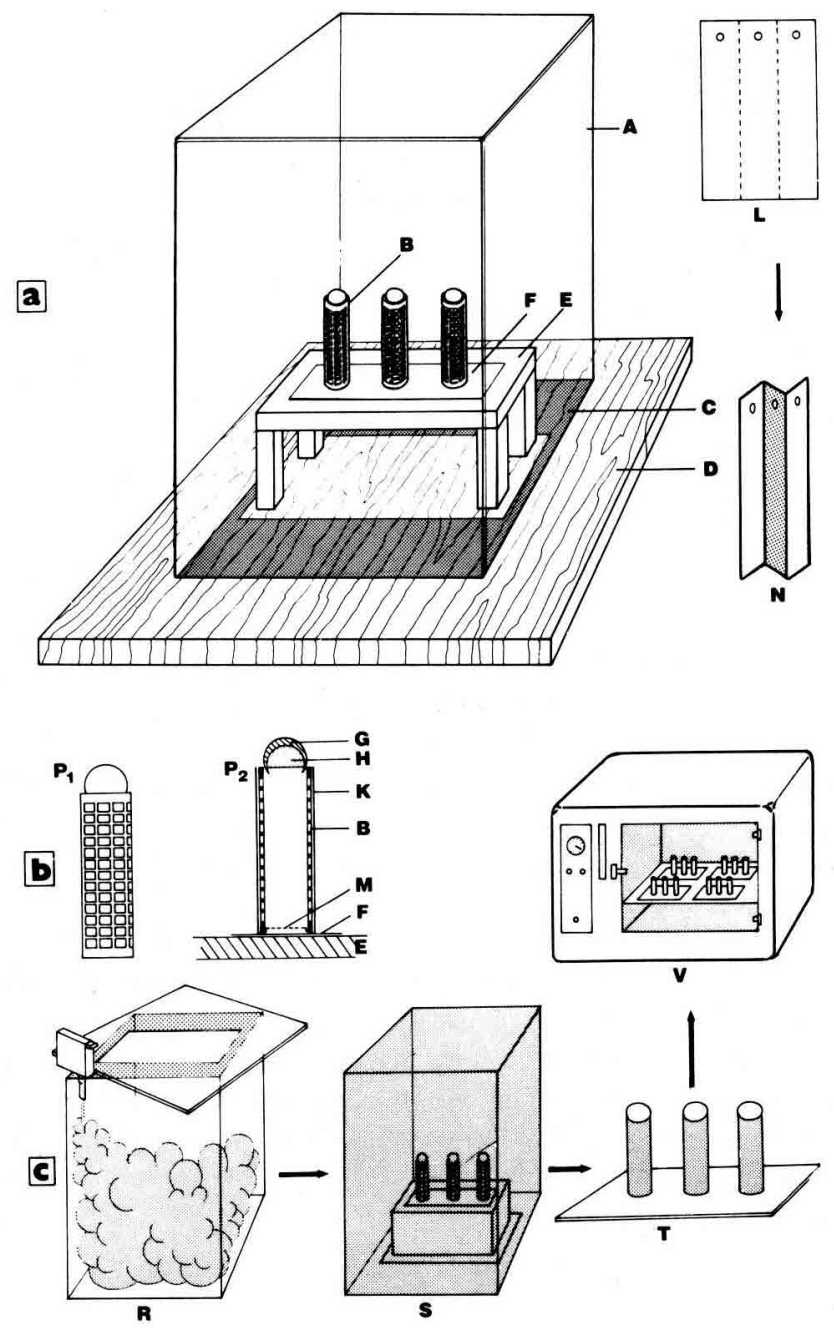

AвB. 1.

a; - Versuchsmaterial und -aufstellung.

A : Kasten aus Perspex; B : Käfig; C : Kunststoffolie; D : Holzunterlage; E : Untersatz (Höhe : 7 cm);

F : Kartonunterlage; $\mathbf{L}$ : Räucherstreifen; $\mathbf{N}$ : idem, gefaltet.

b. - Der Käfig : $\mathrm{P}_{1}$ : Aussenansicht; $\mathrm{P}_{\Sigma}$ : Längsschnitt : Deckel $(\mathrm{G})$ mit Zuckerteig (H),

Papierhülse (K) und Gazesperre (M).

c. - Behandlungsschema. R : Raucherzeugung; $\mathrm{S}$ : Aufstellung während der Rauchbehandlung;

$\mathrm{T}$ : Käfige mit Papierhülsen; V : Versuchskäfige (mit Papierhülsen) im Brutschrank.

FIG. 1.

a. - Experimental material and set-up.

A : Perspex box; B : cage; C : Plastic foil; D : Wooden pad; E : Socle (height : $7 \mathrm{~cm}$ ); $F$ : Cardboard pad; $L$ : Fumigation ticket; $N$ : idem, folded.

b. - The cage. $\mathrm{P}_{1}$ : External view. $\mathrm{P}_{2}$ : longitudinal section : Cover $(\mathrm{V})$ with candy $(\mathrm{H})$, paper envelope $(\mathrm{K})$ and wire gauze $(\mathrm{M})$.

c. - Treatment design. $\mathbf{R}$ : smoke production. $\mathrm{S}$ : set-up during smoke treatment;

$\mathrm{T}$ : Cages with paper envelope. $\mathrm{V}$ : Cages (with envelope) in the incubator. 


\section{SUMMARY \\ DICOFOL FUMIGATION OF HONEY BEES FOR THE CONTROL OF THE MITE VARROA JACOBSONI}

Fumigation paper wicks each containing $0.5 \mathrm{~g}$ of active substance were made with dicofol and a small amount of $\mathrm{KNO}_{3}$. Using the fumigator after VAN LAERE et al. (1982), bees infested by Varroa mites at least 10 per test cage, 3 cages - were treated with dicofol smoke. Fumigation with $5 \mathrm{mg} \mathrm{dicofol} / \mathrm{dm}^{3}$ yielded a $\mathrm{LD}_{s 0}$ for Varroa mites in about 9 minutes. With $10 \mathrm{mg}$ dicofol $/ \mathrm{dm}^{3}$ all treatments resulted in a 100 per cent destruction of the Varroa parasites in 4 minutes. Using this dose, fumigation times of 2 , $4,8,16$ and 32 minutes appeared to be harmless to the bees. Control treatments with $\mathrm{KNO}_{3}$ caused no mortality of Varroa mites and bees. High smoke concentrations resulted in a typical and immediate reaction of the parasites : they left the bees, so that they were more intensively subjected to the smoke.

\section{LITERATURVERZEICHNIS}

CHOI S. Y. and Woo K. S., 1973. - Studies on the bi .umics of the bee mite, Varroa jacobsoni Oudemans, and its chemical control (I). Research Reports of the Office of Rural Development, Suwon, Korea 15 (Livestock) : 35-43. In Korean : English summary.

Filippov V. V., 1978. - (In russian). Scientific investigations on Varroa disease. Pchelovodstvo (6) 2-3.

Ho K. K., HsU E. L. and AN J. K., 1980. - Chemical control of Varroa jacobsoni. 1. Screening test of acaricides on $V$. jacobsoni and toxicity of 5 acaricides to Apis mellifera. Phytopathologist and Entomologist. 78-83.

RITTER and RUTTNER, 1980. Chemotherapie (Varroa). Allg. dtsch. Imkerztg., (5), 138.

RUTTNER and RITTER W., 1980. - Das Eindringen von Varroa jacobsoni nach Europa im Rückblick. Allg. dtsch. Imkerztg. (5) 130-133.

SKRYPNIK E. I., 1977. - Metody ispytania akaritzidov pri varroatoze. Pchelovodstvo (12) 25.

VAN LAeRE O., 1960. - De bestrijding der bijenacariose bij middel van Kelthane. Meded. Landbouwhogeschool und Opzoekingsstations van de Staat te Gent, 25 (3-4) 1315-1320.

Van Laere O., De Wael L. and VAn De Weghe L., 1982. - Eine Vorrichtung zum medikamentösen Räuchern von Bienenvölkern. Allg. dtsch. Imkerztg. (1), 6-7. 